\title{
Germanica
}

\section{La fabrique du présent dans le roman Vaterjahre de Michael Kleeberg}

Die Werkstatt der Gegenwart in dem Roman Vaterjahre von Michael Kleeberg Manufacturing the Present in Michael Kleeberg's Vaterjahre

\section{Lidwine Portes}

\section{(2) OpenEdition}

Journals

Édition électronique

URL : http://journals.openedition.org/germanica/2662

DOI : 10.4000/germanica.2662

ISSN : 2107-0784

Éditeur

Université de Lille

Édition imprimée

Date de publication : 30 décembre 2014

Pagination : $59-78$

ISBN : 9782913857346

ISSN : 0984-2632

\section{Référence électronique}

Lidwine Portes, "La fabrique du présent dans le roman Vaterjahre de Michael Kleeberg », Germanica

[En ligne], 55 | 2014, mis en ligne le 30 décembre 2016, consulté le 06 octobre 2020. URL : http:// journals.openedition.org/germanica/2662 ; DOI : https://doi.org/10.4000/germanica.2662

\section{(c) Tous droits réservés}




\title{
La fabrique du présent dans le roman Vaterjahre de Michael Kleeberg
}

\author{
Lidwine PORTES \\ Université Bordeaux Montaigne
}

\begin{abstract}
Die „Gegenwart“ ist mein Feind und Gegner. Ich glaube, ich habe eigentlich immer geschrieben, um die jeweilige Lebensgegenwart (in Bernstein) $\mathrm{zu}$ versiegeln, sie zu analysieren, zu transzendieren und damit zu retten bzw. ihr überhaupt Körper zu verleihen. Denn den hat die Gegenwart ja eben naturgemäß nicht ${ }^{1}$.
\end{abstract}

Les interrogations sur le temps marquent indéniablement la période qui, en Allemagne, s'ouvre avec la césure de 1989. La littérature du tournant du millénaire est incontestablement marquée par un intérêt pour le passé (W. Hardtwig 2 ), ce qui conduit M. Herrmann ${ }^{3}$ à proposer le néologisme de Vergangenwart ${ }^{4}$ pour désigner ce phénomène. Ainsi peut-on

1. - Michael Kleeberg, in: Johannes, Birgfeld, Michael Kleeberg im Gespräch, Hannover, Wehrhahn, 2013, p. 78.

2. - Wolfgang Hardtwig, Erhard Schütz, Keiner kommt davon. Zeitgeschichte in der Literatur nach 1945. Göttingen, Vandenhoeck \& Ruprecht, 2008.

3. - Meike Herrmann, Vergangenwart, Erzählen vom Nationalsozialismus in der deutschen Literatur seit den neunziger Jahren, Würzburg, Königshausen\&Neumann, 2010.

4. - „Nahezu alle hier untersuchten Romane machen die Gegenwart als Entstehungszeitraum und Blickpunkt auf die Vergangenheit kenntlich [...]. Ihre Zeitform 
postuler sans crainte que le présent est hanté5 par le passé6. S'il semble exister une expérience du temps spécifique à cette période, la littérature contemporaine apparaît comme le réceptacle de cette expérience de la temporalité. De la saga familiale au roman générationnel, la découverte et la reconstruction d'un passé intrinsèquement entremêlé à l'Histoire $\mathrm{du} \mathrm{XX}^{\mathrm{e}}$ siècle et à ses catastrophes est, à n'en pas douter, une des configurations narratives récurrentes de la littérature allemande contemporaine qui contribue, comme cela a désormais été régulièrement souligné, à la construction d'une Erinnerungskultur, « une culture de la mémoire » ${ }^{7}$.

Michael Kleeberg, qui reconnaît volontiers que « les artistes sont les séismographes de leur époque $»^{8}$, s'en était déjà fait le témoin : dans le recueil Der Kommunist vom Montmartre (1997), la nouvelle Liebes Brüderchen, liebe Schwester représente l'année 1989 comme une «brèche temporelle » 9 donnant l'impulsion à une reconstruction mémorielle. Cette brèche s'incarne quelques années plus tard dans une topographie mémorielle, un no man's land au cœur de Berlin d'où va surgir le passé et le récit contrafactuel, le jardin comme hétérotopie ${ }^{10} \mathrm{du}$ roman Ein Garten im Norden (1998).

Dans ce contexte où le passé ne passe pas, il semblerait donc que la littérature contemporaine s'intéresse étonnamment peu, précisément, à ce qui pourrait ou devrait être son objet, le présent. Le diagnostic du présent opéré par la littérature contemporaine montre un présent souvent tourné vers le passé. Ces dernières années, les démarches tentant de cerner ce qui fait l'actualité de la littérature contemporaine se sont ainsi multipliées, des « poétiques du présent ${ }^{11}$ ont émergé qui tentent de mettre au jour les modalités de ce que nous pourrions appeler

ist die Vergangenwart“, in: Herrmann, Vergangenwart, p. 13.

5. - Arne De Winde, Anke Gilleir, Literatur im Krebsgang: Totenbeschwörung und „memoria“ in der deutschsprachigen Literatur nach 1989, Amsterdam, Rodopi, 2008. 2006.

6. - Jean-François Hamel, Revenances de l'histoire, Paris, Éditions de Minuit,

7. - Aleida Assmann, Der lange Schatten der Vergangenheit, Erinnerungskultur und Geschichtspolitik, München, Beck, 2006.

8. - Michael Kleeberg: „Künstler sind Seismografen ihrer Zeit“, in: Johannes Birgfeld, note 1, p. 92.

9. - Lidwine Portes, « Rien que des fantômes. Poétique de la spectralité dans les romans de Michael Kleeberg, Ein Garten im Norden et Das amerikanische Hospital », in : Du Texte à l'image : Appropriations du passé et engagements au présent, Nancy, Le Texte et l'Idée, 2010.

10. - Harald Tausch, ,Der Garten als Heterotopie. Michael Kleebergs Roman Ein Garten im Norden (1998)“. In: Sabine Schneider, Heinz Brüggemann (Hrsg.), Gleichzeitigkeit des Ungleichzeitigen, München, Wilhelm Fink Verlag, 2010.

11. - Silke Horstkotte; Leonhard Herrmann (Hrsg.), Poetiken der Gegenwart, Deutschsprachige Romane nach 2000, Berlin, De Gruyter, 2013. 
la fabrique du présent. Quels sont les marqueurs spécifiques du hic et nunc ? S'agit-il des thèmes, dans une veine réaliste, qui contribuent à ébaucher une peinture de la société du XXI ${ }^{\mathrm{e}}$ siècle ? Si oui, s'agit-il finalement toujours de ramener la littérature à l'écriture de l'Histoire - fûtelle immédiate - ou faut-il chercher les caractéristiques de la littérature du présent du côté de modalités esthétiques qui tenteraient peut-être de dépasser le postmodernisme?

Le roman Vaterjahre, paru en août 2014, est un roman du présent qui s'inscrit de manière tout à fait singulière dans la création contemporaine. Dans le contexte d'une littérature allemande dominée par la vague mémorielle, Michael Kleeberg offre au lecteur un roman qui construit un artefact du temps présent, dans lequel converge la totalité du monde. Si l'auteur définit le " présent comme le laps de temps vécu par des personnes vivantes avec lesquelles je peux parler directement, comme la période que je peux saisir parce que j'en ai entendu parler », il conclut de manière un peu provocatrice que « le présent [...], pour le dire vite, englobe pour moi tout le $\mathrm{xx}^{\mathrm{e}}$ siècle $»^{12}$. C'est pourtant bien le présent du tournant du millénaire que le roman Vaterjahre (2014) capture dans sa texture polyphonique et composite, tendant un miroir à la société allemande du XXI ${ }^{\mathrm{e}}$ siècle, marquée par la globalisation et par une expérience spécifique de la temporalité : entre catastrophes collectives et individuelles, le récit emporte le lecteur dans vingt-quatre heures d'un présent dilaté qui débouche sur un panorama du $\mathrm{xx}^{\mathrm{e}}$ siècle.

\section{Capturer le présent - le roman comme artefact du temps}

On retrouve dans Vaterjahre le protagoniste qu'on avait quitté à la fin du roman Karlmann (2007), Charly Renn, et qu'on avait suivi dans ses années de jeunesse, de 1985 à 1989, du jour de son mariage avec Christine, assistant à ses infidélités et à la fin de leur union. Dans cette deuxième partie du cycle romanesque entrepris par Michael Kleeberg, on le retrouve père de deux enfants. Quelque chose de fondamental a changé, il n'est plus l'homme autocentré qu'il était. Depuis la naissance de ses enfants, la peur est entrée dans sa vie ${ }^{13}$ et son rapport au temps

12. - Michael Kleeberg: „Ich definiere für mich Gegenwart als den Zeitraum, den lebende Menschen erlebt haben, mit denen ich mich noch direkt darüber austauschen konnte, den Zeitraum, den ich vom Hörensagen umfassen kann. Gegenwart (sofern wir sie nicht als den zerstäubenden Augenblick des jetzigen Moments definieren, der für die Literatur ultraviolett, also nicht darstellbar ist) umfasst für mich also kurz gesagt das gesamte 20. Jahrhundert", in: Johannes Birgfeld, note 1, p. 35.

13. - Michael Kleeberg, Vaterjahre, p. 12: „Was hat sich entscheidend und unumkehrbar verändert seit den Geburten, seit der ersten Geburt, dann wäre die Antwort : Angst ist in dein Leben getreten". 
a changé. Il archive désormais le présent comme « une photo-souvenir de l'instant ${ }^{14}$.

Die entscheidende Frage bei Karlmann ist weniger, wie lange die Geschichte zeitlich reichen soll, sondern welche Bedeutung die vergehende Zeit für diese Geschichte hat: eine ganz entscheidende nämlich bezogen sowohl auf die Individuen wie auf die gesellschaftlichen Aspekte. Die Zeit verlangt also einen anderen formalen Zugriff als im ersten Teil, einfach deswegen, weil sie, vergehend, für die Protagonisten wie für den Autor, anders wahrgenommen wird und daher andere Konsequenzen zeitigt. Die Zeit wird sich in alle Richtungen ausdehnen und -breiten, nicht nur in die Zukunft, sondern eben auch in die Vergangenheit hinein ${ }^{15}$.

Alors que la diégèse de Karlmann s'appliquait à éclairer la vie du personnage en mettant en lumière quelques heures tirées de quelques journées prises sur cinq années, celle de Vaterjahre condense le récit sur une durée de 24 heures : le roman s'ouvre sur une scène qui sera clôturée quelques 500 pages plus loin, le lendemain soir. Parce que la chienne, Bella, est très malade, la scène du coucher des enfants, qui ancre d'emblée la diégèse dans le quotidien, est marquée par l'incursion brutale et prématurée de la mort dans la vie ordinaire de cette famille. Le roman se termine le lendemain soir par une scène familiale après que le $\mathrm{D}^{\mathrm{r}}$ Bielefeldt est venu abréger les souffrances de l'animal, semblant ainsi inscrire le roman dans un continuum temporel présent. De ce point de vue, le temps de l'histoire recouvre les 24 heures de la vie ordinaire d'un homme, Charly Renn, aux prises avec un événement familial tragique, entraînant la nécessité de trouver un récit acceptable pour une enfant de six ans.

Mais entre ces deux chapitres viennent s'insérer 4 autres chapitres qui font un usage foisonnant d'analepses et - fait certainement plus rare pour le roman - de prolepses ${ }^{16}$. Les chapitres $2,3,4$ et 5 remontent le temps de l'année 1993 à 2000 et éclairent tour à tour des aspects de la vie professionnelle, amoureuse et amicale de Charly, donnant corps aux réflexions du personnage sur le temps, qui apparaissent comme des clins d'œil métadiégétiques. Charly considère en effet « qu'il est arrivé à un âge où le présent a cessé d'être la temporalité la plus importante ${ }^{17}$.

14. - Michael Kleeberg, Vaterjahre, p. 85 : „Erinnerungsfoto des gegenwärtigen Augenblicks“.

15. - Michael Kleeberg, in: Johannes Birgfeld, note 1, p. 95.

16. - Voir l'épisode le plus marquant: Michael Kleeberg, Vaterjahre, p. 247 : „Wir greifen als vor in den Sommer 2002".

17. - Michael Kleeberg, Vaterjahre, p. 91 : „daß er in einem Alter angekommen ist, in dem das Präsens aufgehört hat, die wichtigste Zeitform zu sein“. 
Das Leben, das noch wenige Jahre zuvor hic et nunc war, ist nun ein zäher Kaugummi, dessen eines Ende irgendwo und irgendwann in der Vergangenheit festgeklebt ist und dessen anderes Ende deine Kiefer immer noch kauen, mag auch aller Geschmack daraus verschwunden sein; dazwischen aber hängen Schlieren und dünne, feuchte, graue Hängebrücken in der Luft - erstaunlich haltbar und stabil, und was immer gerade geschieht, hat eine schleimige Verbindung zurück ins Labyrinth der Vergangenheit an diesem klebrigen Ariadnefaden entlang. (VJ, 135-136)

Ces chapitres apparaissent donc comme autant de haltes significatives dans le labyrinthe du passé.

Si la structure du roman paraît offrir quelque progression chronologique, la numérotation des chapitres sape pourtant la notion même de progression. Les six chapitres du roman voient revenir les numéros et les titres des trois premiers chapitres dans un ordre différent ${ }^{18}$. Cette numérotation remet en question la foi en la progression de la construction romanesque : Charly Renn semble prisonnier de cette triade que forment la vie privée, le travail et l'entourage, puisque tels sont les noms des chapitres qui reviennent en boucle. Il semblerait qu'arrivées à la fin du troisième chapitre, toute progression, toute évolution soient exclues. Le péritexte invite ainsi, avec cette singulière numérotation qui piétine dans une temporalité répétitive et stagnante, à chercher le sens du roman non dans la progression d'une intrigue ni dans l'évolution d'un personnage, mais ailleurs, comme si Michael Kleeberg signalait ainsi à son lecteur son intention d'« écrire un roman sans intrigue ou plutôt un roman, dans lequel l'essentiel ne serait pas l'intrigue, mais la langue et la forme - en d'autres termes le fameux "roman sur rien" de Flaubert »19.

Michael Kleeberg excelle en effet, dès l'ouverture du premier chapitre, dans l'art de recréer, au niveau de la narration, la texture du temps présent. Le lecteur se retrouve plongé in medias res dans les pensées de Charly qui observe sa fille Lulu, endormie. Ses pensées sont tour à tour présentées par le truchement d'une focalisation interne, puis d'une focalisation zéro. Des bribes du dialogue entre le père et sa fille alternent avec le style indirect libre ou des remarques du narrateur omniscient, multipliant ainsi non seulement les points de vue sur Charly, mais variant aussi les modes narratifs et ainsi la distance. Le temps de

18. - Les chapitres se succèdent dans l'ordre suivant: Kapitel 1 - Privatleben; Kapitel 2 - Arbeit; Kapitel 3 - Umfeld; Kapitel 1 - Privatleben; Kapitel 3 - Umfeld; Kapitel 2 - Arbeit.

19. - Michael Kleeberg: „Der Wille, [...] einen Roman ohne Plot oder vielmehr einen Roman, in dem der Plot nicht mehr das Movens ist, sondern die Sprache und die Form - kurzgesagt Flauberts ominösen ,Roman über nichts'“, in: Johannes Birgfeld, note 1, p. 80. 
l'histoire ainsi étiré ne tarde pas à être élargi à celui du souvenir avec des analepses qui conduisent Charly dans « une pièce éclairée dans la maison hantée de la mémoire dont il ignorait l'existence : la pièce où sont consignés les souvenirs $\gg^{20}$. Poursuivant les expériences narratives entreprises dans Karlmann, Vaterjahre repousse les limites des possibles en multipliant les visages de l'instance narrative et s'applique à mettre en place une troublante polyphonie. Ainsi a-t-on des glissements permanents, parfois dans le même paragraphe, parfois dans une même phrase, entre focalisation zéro et interne, entre narration à la deuxième et à la troisième personne du singulier. L'instance narrative démultipliée émane tour à tour du $t u$, du $i l$ et du nous. La nouveauté étant peut-être que le narrateur, qui, comme le souligne Johannes Birgfeld, se tenait en retrait dans Karlmann ${ }^{21}$, sort ici de sa réserve et affirme au contraire son individualité et son autorité. Ses interventions marquent parfois sa supériorité, lorsqu'il souligne par exemple que les mots manquent à Charly face à la beauté de sa fille, une remarque qui peut paraître légèrement ironique insérée au cœur d'une minutieuse description de l'enfant. Ce narrateur se caractérise dès l'incipit comme le « Janus de cette histoire, qui regarde dans le futur et dans le passé, son visage dialectique à deux faces $»^{22}$. Il brise l'illusion fictionnelle lorsqu'il partage avec le lecteur ses interrogations autoréflexives sur la suite à donner à un épisode du récit ou lorsqu'il ajoute une information sur Kumpf, qui sort du cadre temporel dans lequel la diégèse est ancrée.

Ironischerweise ist genau das beinahe geschehen, einige Jahre später, was uns allerdings zwingt, aus dem Zeitrahmen unserer Geschichte hinauszutreten. Es gehört sich zwar eigentlich nicht, zeitlich über die Ränder eines Buches hinauszugreifen und Dinge aus einer hypothetischen Zukunft in die erzählte Zeit hereinzuholen, in der sie keine Rolle spielen, weder erwartet noch erhofft sind und außerhalb jeder Realität und Vorstellbarkeit für die handelnden Personen [...]. (VJ, 247)

Ainsi ne peut-on oublier sa présence, même lorsque les pensées de Charly sont livrées sans guillemets et que le je, par moments, semble s'émanciper et être le marqueur du flux de conscience, car l'alternance est permanente, les interpellations de l'instance narrative à un narrataire

20. - Michael Kleeberg, Vaterjahre, p. 20: „eine hell ausgeleuchtete Asservatenkammer der Erinnerung, von deren Existenz im Spukhaus des Gedächtnisses er gar nichts ahnte“.

21. - Ainsi Johannes Birgfeld souligne-t-il à propos de Karlmann que : « Mit Auskünften über sich selbst, mit Wertungen, die den Erzähler als Person profilieren würden, hält dieser sich zurück » (Johannes Birgfeld, Eine Werksbegehung, DVA, 2014, p. 248).

22. - Michael Kleeberg, Vaterjahre, p. 9: ,,der vorans- und zurückblickende Janus dieser Geschichte, ihr dialiektisches Doppelgesicht". 
à la deuxième personne du singulier, constantes, et les glissements entre ces différentes positions, permanentes.

Les interpellations à la deuxième personne du singulier induisent ainsi une dimension dialogique. Dans certains passages, le lecteur se demande s'il a affaire à un dialogue de Charly avec lui-même, comme ici lorsque Charly contemple la beauté de sa fille :

Und würdest $d u$ sie wirklich weniger lieben, hielte sie deinen Ansprüchen nicht stand? Also enttäuscht wäre ich schon. (VJ, 16)

Qui est ici le je qui répond à la question ? S'agit-il du narrateur qui partage ses réflexions sur le personnage ou de Charly qui serait déçu si sa fille n'était pas à la hauteur de ses propres exigences ? L'instance narrative procède également du $t u$ pour livrer les souvenirs de Charly (du erinnerst dich, wie...), ses impressions après-coup (so kam es dir vor, VJ 17), donnant corps au « point de vue après les faits $»^{23}$ décrit par Monika Fludernik. Mais le $t u$, par-dessus tout, contribue à la déstabilisation du lecteur. Ainsi au chapitre 4 (alias Kapitel 1 : Privatleben, p. 259), le point de vue est homodiégétique dans un récit alternant première et deuxième personne du singulier. Mais cette fois, l'histoire est présentée du point de vue de Heike, l'épouse de Charly, et l'adresse à la deuxième personne du singulier apparaît tour à tour comme un dialogue de Heike avec elle-même et comme une adresse du narrateur à Heike. Ainsi voit-on s'opérer le glissement, comme dans le passage suivant où la dimension métadiégétique illustre l'ambition multiperspectiviste du roman :

Charly insistiert nie. Er interessiert sich für die Orte und die Freunde und die Familie, aber nicht für die Männer vor ihm. So wie ich mich nicht für die Frauen vor mir interessiere. Erschreckend, wenn du dich erinnerst, ist lediglich, dass man währenddessen immer nur die eine Seite sieht, auf die das Licht fällt. (VJ, 304)

En déplaçant la focalisation de Charly à Heike, le roman affiche son ambition totalisante, mais également sa conscience d'un sujet insaisissable dans sa totalité, ce que semble confirmer ces remarques du narrateur/de Heike quelques lignes plus bas :

23. - « un after-the-fact-point of view », in : Monika Fludernik, « Second-person narrative as a test case for narratology », Sonderdrucke aus der Albert-LudwigsUniversität Freiburg, Originalbeitrag erschienen in : Harold F. Mosher (Hrsg.) : Secondperson narrative. Dekalb, Ill.: Northern Illinois Univ., 1994. (Style; 28,3) S. 445-479, p. 449. 
Lektion ist, sich selbst zu misstrauen in seiner Genügsamkeit, nur eine Facette des anderen sehen zu wollen unter Ausblendung aller anderen. (VJ, 304)

Ainsi la narration à la deuxième personne du singulier semble-t-elle significative de la constitution intersubjective du sujet, tant on hésite entre les différentes interprétations à donner à ce $t u$ : dialogue de Charly avec lui-même, soliloque dans l'après-coup ${ }^{24}$, ou une interpellation du narrateur à son narrataire-personnage Charly ${ }^{25}$, on assiste en tout cas à un glissement permanent qui permet de faire passer le point de vue du niveau individuel au niveau exemplaire. La prouesse narrative de Vaterjahre semble mettre en abyme la nature instable du sujet et interroger la possibilité d'en faire le récit. L'alternance des voix narratives permet indéniablement de changer de plan en permanence et de passer de l'expérience individuelle à l'expérience universelle, comme l'illustre le récit de l'accouchement de Heike où la position de Charly est élargie à celle de Joseph. La capture du présent semble s'opérer dans ce moment de la naissance, immortalisé par les tableaux de la Renaissance dans les portraits de la Vierge à l'enfant :

[...] der Blick, in dem sich Staunen, Bangigkeit und zärtliche Zuneigung die Waage halten, scheint über die festgehaltene Sekunde hinaus in eine Art von Ewigkeit zu gehen, in ein Vergangenheit und Zukunft umfassendes Präsens [...]. (VJ, 35)

De la même manière que la polyphonie des voix permet d'éclairer des facettes restées dans l'ombre, le roman semble être un réseau dans lequel il est possible de naviguer, constitué d'événements proches et lointains, qui, selon la perspective, sont montrés en gros plan ou en plan large. On assiste à un jeu de réglage permanent de la caméra qui ne cesse d'ajuster et de réajuster son focus, en assumant d'être vue du lecteur. Lorsqu'il s'agit par exemple de prouver que Charly ne souffre pas d'un Edipe mal réglé, l'instance narrative n'hésite pas à faire un bond sur la carte géographique de ses personnages et à déplacer le lieu de l'action et de l'histoire à Arcachon pour prouver l'exactitude d'une information :

Zum Beweis, daß jeder freudianische Interpretationsversuch hier fehl am Platz ist, blicken wir, ohne dabei Charly aus dem Auge zu verlieren, eben in eine Entfernung von 1279 Kilometern und betrachten das Ganze von der anderen Seite aus : In jenem September 1993 befand

24. - « an internal memory monologue in the second person », in : Fludernik, Ebd., p. 449.

25. - Une variante « homocommunicative » de Fludernik, où « the narratee is an actant and the narrator merly a voice on the communicative level », in : Fludernik, Ebd., p. 448. 
sich Karl Renn mit seiner Frau in ihrem im Vorjahr auf eine, wie Charly das nannte : Schnapsidee hin gekauften Ferienhaus bei Arcachon. (VJ, 92)

La narration apparaît comme un vaste réseau de connaissances qui n'est pas sans rappeler l'internet, le geste du narrateur montrant qu'il est possible de naviguer au sein du roman comme au cœur d'un document hypertexte. D'ailleurs, le récit, afin d'éclairer les différentes facettes de son personnages central, fait appel à différentes sources sans s'en cacher : journaux intimes ou calendriers familiaux sont intégrés dans le corps du texte. Le roman semble être une mise en abyme de la réalité enrichie. Toutes les informations sont quelque part à disposition. L'intermédialité ici à l'œuvre contribue à la construction d'une vision totale. Elle permet de faire converger la totalité dans le présent.

Ich bilde mir ein, und die Fortschreibung des KarlmannProjektes wird es erweisen müssen, dass mir die Entwicklung dieses Erzählplasmas ermöglichen wird, die vorgenommene und erhoffte Totalität, den Totalzugriff auf die Welt, wenn nicht zu erreichen, dann doch ein ganzes Stück weiter zu treiben, als mir das bisher möglich $\operatorname{war}^{26}$.

Ainsi la fabrique du présent s'élabore-t-elle sur tous les fronts du roman : péritexte (à travers la numérotation répétitive des chapitres) et texte (à travers le multiperspectivisme) concourent à désigner le roman comme un roman sur le temps, comme si Michael Kleeberg parvenait ici à construire un artefact du temps, conférant à son écriture les qualités suivantes :

Insofern wäre mein Schreiben ein permanenter Prozess von Gegenwartsverfestigung und Archivierung, nicht etwa thematisch, sondern substantiell und strukturell gesehen, als die Essenz all dessen, was ich zu bestimmten Momenten als Lebens- und Literaturreuse in mir getragen habe 27 .

\section{Gegenwärtigkeit : les marqueurs du hic et nunc}

Cette fabrique du présent ne s'élabore pas uniquement sur le plan narratif et formel, elle est également, pour emprunter le terme à Roland Barthes, le fruit d'un effet de réel ${ }^{28}$ qui ancre le présent avec lequel

26. - Michael Kleeberg, in: Johannes Birgfeld, note 1, p. 84.

27. - Johannes Birgfeld, note 1, p. 78.

28. - Roland Barthes, «L'effet de réel », in : Roland Barthes, Le Bruissement de la langue, Paris, Seuil, 1984. 
Charly Renn est aux prises, dans une société allemande post-réunification, profondément globalisée. Si le continuum présent est ancré dans les deux journées des 10 et 11 septembre 2001, les analepses éclairent les années 90.

L'arrière-plan sur lequel Charly perçoit l'ensemble du monde est la ville hanséatique d'Hambourg :

[...] das war Hamburg: seine kühne Gediegenheit, seine solide Freibeuterei, seine honorige Chuzpe, seine himmelstürmende Bodenständigkeit und seine ästhetische Kaufmannsklugheit. (VJ, 209)

Cet ancrage d'Hambourg dans la tradition s'incarne dans l'entreprise patricienne de Jessen que Charly rejoint dans le prestigieux bâtiment du Chile-Haus. Hambourg et son architecture apparaissent comme l'un des derniers bastions de « la qualité, de la beauté, des détails, de la solidité et de la durée ${ }^{29}$ dans un monde globalisé où les objets et les constructions sont standardisés et immédiatement désuets. Cette conscience d'une autre foi en l'avenir est un rempart face à l'entropie du présent et à « notre modernité désespérée et sans mémoire » ${ }^{30}$.

La peinture du présent est dominée par l'omniprésence du monde économique. Charly Renn évolue dans le milieu des cadres d'entreprise performants. Il perçoit les rapports humains à l'aune des tableaux de pertes et de profits, lorsqu'il évoque sa relation amoureuse par exemple, il considère que son épouse, «Heike[,] a investi en [lui] lorsque l'action était au plus bas. C'est logique que ses dividendes augmentent $\gg^{31}$. Lorsque Charly tente de cerner les ressorts de sa rivalité avec son meilleur ami Kai, il perçoit leurs différences de parcours à travers le prisme de la rentabilité. Charly pense avoir perdu son temps, alors que Kai en a fait quelque chose. La réalité de Charly Renn semble marquée par une économisation des rapports humains. Cette dernière va de pair avec l'omniprésence, dans le roman, d'une langue économique qui est celle de la spéculation boursière. Mais Charly Renn, tout performant qu'il soit, a un accident de parcours : suspendu un instant dans les cieux sur la prouesse architecturale qu'est le pont Köhlbrandbrücke, il est contraint par ses entrailles de s'immobiliser dans cet entre-deux, et touche littéralement le fond, vivant la pire humiliation de son existence. Afin de surmonter cette crise, il se rend chez Petra Wedekind, psychologue-

29. - Michael Kleeberg, Vaterjahre, p. 212: „Qualität, Schönheit, Details, Solidität und Dauerhaftigkeit".

30. - Michael Kleeberg, Vaterjahre, p. 212: ,unserer heillosen, erinnerungslosen Modernität“.

31. - Michael Kleeberg, Vaterjahre, p. 130 : „Heike hat in mich investiert, als die Aktie auf dem Tiefststand war. Logisch, dass das dann zu einer Dividendenausschüttung führt". 
comportementaliste, et y déroule le tableau de sa vie professionnelle. Sur son divan, Charly doit en permanence traduire le jargon qui fait son quotidien en des termes accessibles au commun des mortels. Ainsi explique-t-il à cette intellectuelle les codes du monde de l'entreprise. Il l'initie aux règles prévalant au management des coûts et des ressources humaines et lui explique que dans le monde de la recherche permanente du profit, « il n'y a pas de facteurs mous. Pas d'individualité. Si Madame X ne fait pas ça en trois minutes, elle n'est pas à sa place ${ }^{32}$. Le milieu de l'entreprise constitue la grille de lecture du réel de Charly, aux antipodes de celui de la psychologue. À cette époque, Charly, qui a fait des études d'économie politique, est encore directeur commercial d'un courtier maritime, au moment où l'entreprise fait appel à l'expertise du cabinet McKinsey, dont le rôle est d'« augmenter les profits [...] [ou de] redresser les comptes $»^{33}$. Le cabinet McKinsey représente le secteur dit de l'accompagnement du changement. Propre à la période de crise, il encourage les entreprises à être flexibles et à opérer des mutations auxquelles les salariés doivent s'adapter. De la même manière que Michael Kleeberg avait procédé à de minutieuses recherches pour aborder les sujets techniques de la reproduction médicalement assistée et de la guerre moderne dans Das amerikanische Hospital, Vaterjahre plonge les lecteurs dans les rouages de l'économie d'entreprise. Ainsi peut-on suivre Charly, désigné par sa direction pour être le manager du changement, en trois semaines de séminaires dans un centre de formation pour cadres dirigeants sur « les fondements de la gestion de processus et de la médiation ${ }^{34}$. De retour, Charly ne peut s'empêcher d'appliquer ses nouvelles compétences à sa vie privée : «Si j’avais maîtrisé ces techniques à la fin de mon premier mariage, je me serais épargné pas mal de stress $»^{35}$. Les techniques managériales de l'entreprise sont la modalité avec laquelle le personnage de Vaterjahre appréhende la réalité. Laissant loin derrière lui la crise de la Köhlbrandbrücke, il est devenu un homme mûr, sûr de lui, qui contrôle ses émotions et les rapports humains grâce aux compétences sociales et communicationnelles qu'il a acquises. Pour reprendre la métaphore qui traverse le roman, la fontanelle sociale est refermée $^{36}$. Sa vie est parfaitement compartimentée : il sait «évaluer ce

32. - Michael Kleeberg, Vaterjahre, p. 134. „,...] existieren keine Softfacts. Keine Individualität. Wenn Frau X das nicht in drei Minuten schafft, dann ist sie fehl am Platze“.

33. - Michael Kleeberg, Ebd., p. 133 : „entweder, um die Erträge zu verbessern, oder um zu sanieren“.

34. - Michael Kleeberg, Ebd., p. 155: „'Grundlagen des Prozessmanagements’ und 'Mediation'".

35. - Michael Kleeberg, Ebd., p. 157: „Hätte ich diese Technik schon am Ende meiner ersten Ehe beherrscht, wäre mir viel Stress erspart geblieben“.

36. - Michael Kleeberg, Ebd., p. 207-208: „Verknöcherung der sozialen 
qui le concerne personnellement, ce qui ne concerne que son entourage et ce qui ne le regarde absolument pas ${ }^{37}$. L'économie des émotions développée par Charly est mécanique :

Sie befähigt einen umzuschalten (ruckfrei wie ein gutes Automatikgetriebe, das zugleich auch den Emotionsverbrauch senkt) zwischen Betroffenheit, Anteilnahme, Interesse, Desinteresse, Höflichkeit, der Verfolgung eigener Interessen und altruistischen Nischen. (VJ, 206)

Doté de ce sens aigu de la compartimentation, Charly n'est ainsi que moyennement affecté par le destin de Jobst, un de ses amis de jeunesse. Aux obsèques de la tante Yvonne, les écarts de parcours entre les deux hommes paraissent déjà des distances infranchissables. Charly perçoit son ami comme un « raté » ${ }^{38}$. Suite à des problèmes professionnels, la mécanique du décrochage social semble s'être enclenchée dans sa vie : perte d'emploi, baisse de niveau de vie, divorce... Jobst, dans sa descente aux enfers, est mis au ban de la bonne société hambourgeoise rassemblée ce jour-là et semble aux antipodes de l'univers du tout Hambourg incarnée par Henriette von Bismarck ou par la pasteur qui gère sa carrière comme un personnage médiatique.

À l'instar de l'individu qui atteint l'âge mûr en développant cette aptitude à l'égoïsme, la société allemande du tournant du millénaire dans laquelle Charly Renn évolue avec aisance, est une société profondément compartimentée, comme l'illustre la peinture de Berlin, pauvre et multiculturel. Alors que Kai et Charly sont invités à visiter la très cossue villa d'Inès qui s'est remariée, l'écart entre deux mondes devient flagrant. Les deux amis traversent Berlin en voiture et sont frappés par la pauvreté qui tranche avec l'ordre et le luxe d'Hambourg. Alors qu'en « linguistes et sémioticiens aguerris de leur ville natale »39, ils maîtrisent les codes leur permettant de donner sens à l'espace hambourgeois, leurs impressions de Berlin produisent $«$ une image disparate ${ }^{40}$. La nouvelle capitale allemande, « une ville qui vit de subventions »41, leur fait l'effet des « villes industrielles sibériennes de l'après-guerre » ${ }^{42}$. Évoqué sur le mode de la parataxe et de l'enchaînement de phrases nominales au

Fontanelle“.

37. - Michael Kleeberg, Ebd., p. 206: „Sie lehrt einen gradieren in das, was einen persönlich betrifft, was nur Umfeld ist und was einen gar nichts angeht".

38. - Michael Kleeberg, Ebd., p. 199: „Versager“.

39. - Michael Kleeberg, Ebd., p. 359 : „Linguisten und Semiotiker ihrer Heimatstadt".

40. - Michael Kleeberg, Ebd., p. 360 : ,ein disparates Bild“.

41. - Michael Kleeberg, Ebd., p. 362 : ",ne subventionierte Stadt".

42. - Michael Kleeberg, Ebd., p. 361: „Schwarz-Weiß-Film über sibirische Industriestädte der Nachkriegszeit“. 
rythme saccadé, le paysage urbain berlinois représente la rupture, la juxtaposition désordonnée d'éléments, comme après le passage d'un cyclone, ne permettant pas l'émergence du sens.

Nirgendwo etwas Erhabenes, nichts Auffälliges. Nichts Vertikales, nichts Grandioses, kein Überblick, keine Durchbrüche $\mathrm{zu}$ einer Panoramaaussicht, keine kühn sich schwingenden Bögen (wie die Köhlbrandbrücke!), keine Reliefs, eine Ebene, eine endlose städtische, halbstädtische Ebene, durch die ein Wirbelsturm gebraust war, der alles vom Zentrum weggeschleudert hatte, und alles war liegengeblieben, wo es hingefallen war, willkürlich, übereinander, durcheinander, Steine, Blech, Haut und Fleisch, Bäume - es war wie der Sturm am Anfang des Zauberers von $\mathrm{Oz}$. (VJ, 361)

De la même manière que tout oppose les deux villes de Berlin et d'Hambourg, tout semble désormais séparer le monde de Jobst de celui d'Inès. Inès habite une villa à Potsdam séparée de l'extérieur par une clôture qui entoure quatre maisons gardées par un service de sécurité et par un système de vidéo-surveillance dernier cri. Elle vit avec Volker, qui promeut l'initiative privée « là où les communes et où le service public échouent pour des raisons structurelles ou idéologiques ${ }^{43}$ et qui considère «que notre société est en train de retourner à l'état du $\mathrm{XIX}^{\mathrm{e}}$ siècle ${ }^{44}$. Jobst, l'intrus, « le vagabond bourré » ${ }^{45}$, qui n'a plus les moyens d'aller chez le dentiste, dérange sa nouvelle vie. Dans la nuit du 28 au dimanche 29 octobre, Jobst meurt de froid sur un banc, après avoir rendu une dernière visite à la bande d'amis, qui, à l'instar de Charly passé maître dans l'art de gérer ses émotions -, choisissent de ne pas se laisser troubler par les « mauvaises vibrations »46 émanant de celui qui ne fait plus partie de leur monde et se débarrassent de lui en lui glissant un billet de 100 DM.

\section{Histoire du présent - Présence de l'Histoire}

Si la peinture de la société allemande des années 1990-2000 éclaire le présent, le roman Vaterjahre n'en joue pas moins avec les codes littéraires de son époque et aborde le topos du passé familial, paradigmatique de la littérature allemande du tournant du millénaire. Le présent dilaté débouche sur un panorama du $\mathrm{Xx}^{\mathrm{e}}$ siècle de la Première Guerre mondiale au 11 septembre 2001.

43. - Michael Kleeberg, Ebd., p. 383-384 : ,wo die Kommunen und die öffentliche Hand aus strukturellen, persönlichen oder ideologischen Gründen versagen“.

44. - Michael Kleeberg, Ebd., p. 384 : ,dass wir gesellschaftlich auf dem Weg zurück ins neunzehnte Jahrhundert sind“.

45. - Michael Kleeberg, Ebd., p. 370 : „,der besoffene Landstreicher“.

46. - Michael Kleeberg, Ebd., p. 375: „,bad vibrations“. 
Pourtant Vaterjahre se distingue nettement des romans familiaux qui occupent l'espace littéraire entre 1995 et 2005. En novembre 1998, toutes les générations de la famille Renn entreprennent un voyage en Belgique pour se rendre sur la tombe du grand-oncle de Charly au cimetière militaire allemand de Langemarck. Au moment des commémorations du centenaire de la Première Guerre mondiale, le roman semble adopter un instant les codes de la littérature de la mémoire. Ce voyage dans le passé de la bataille d'Ypres où de nombreux soldats perdirent la vie doit servir de caution morale au présent.

Man darf sich fragen, inwieweit die Idee, das Grab eines Mannes zu besuchen, der im Ersten Weltkrieg gefallen und von dem in der Familie noch nie die Rede gewesen war, ursächlich zu tun hatte mit den in diesen Wochen in Hamburg aufkommenden Fragen nach dem Bruder dieses Toten. Ein grüner Abgeordneter [...] bemühte sich aber nach Kräften, einen Skandal daraus zu machen, pünktlich zum sechzigsten Jahrestage der Pogrome, und zwar aus der Frage, ob das Juweliergeschäft Renn arisiert worden bzw. ob es beim Verkauf durch den jüdischen Vorbesitzer an Großvater Renn im Jahre '38 juristisch und moralisch mit rechten Dingen zugegangen war. (VJ, 243)

De la même manière que Karlmann livrait « un portrait des années $80 »^{47}$, ici ce sont les réalités allemandes du tournant du millénaire qui forment le quotidien de Charly Renn. Elles sont marquées entre autres par le règlement des affaires de spoliations liées aux années de nazisme. Cette affaire est évoquée comme un détail banal. La mémoire est transgénérationnelle et dialogique, puisque les interprétations du passé sont plurielles et divergentes selon les nations, comme l'illustre ici l'échange entre le guide belge et la famille allemande. Si ce lieu de mémoire consacre les souffrances de «tout un continent traumatisé ${ }^{48}$, la mémoire reste pourtant bien individuelle et c'est via l'individu et son « cordon ombilical généalogique et historique ${ }^{49}$ que la totalité de l'Histoire du $\mathrm{Xx}^{\mathrm{e}}$ siècle converge tout naturellement dans le présent. C'est d'ailleurs la même mémoire dialogique qui vient inscrire les 40 ans de RDA dans le présent de Charly Renn, lorsqu'au détour d'une conversation qui porte les marqueurs du quotidien et de l'ordinaire, on apprend que Heike, sa femme, vient de RDA. Là encore, cela est évoqué de manière anecdotique à travers le regard d'un homme

47. - „Porträt der achtziger Jahre“, Branka Schaller-Fornoff, „Fermentierungen des Pop in Michael Kleebergs Karlmann“. In: Katja Kauer (Hg.): Pop und Männlichkeit. Zwei Phänomene in prekärer Wechselwirkung ?, Berlin, Frank\&Timme, 2009, p. 105118 , p. 116.

48. - Michael Kleeberg, Vaterjahre, p. 255: „Ein ganzer traumatisierter Kontinent“.

49. - Michael Kleeberg, Ebd., p. 251. 
qui « a toujours détesté la RDA, d'Ulbricht à Stecher et Sparwasser jusqu'à Guillaume » ${ }^{50}$, qui considère les citoyens de RDA comme « les Allemands auxquels les Russes ont fait un lavage de cerveaux. Les faux Allemands $»^{51}$, qui exècre l'Ostalgie permanente à laquelle se livrent les membres de sa belle-famille, qui se remet difficilement de ce que sa « femme soit la fille d'un sbire du SED »52 et qui, par-dessus tout, déteste l'odeur de la RDA, « cette odeur de phénol qui, dans cet appartement, transpire des murs et du sol et qu'on prend d'abord pour l'odeur de renfermé de RDA ${ }^{53}$. Vaterjahre est donc un roman résolument allemand, qui aborde la grande Histoire par le petit bout de la lorgnette du regard individuel et subjectif de Charly Renn. Le panorama du $\mathrm{Xx}^{\mathrm{e}}$ siècle à travers la peinture des années post-réunification n'en est que plus truculent :

Ach, Schwamm drüber, sagt seine Schwiegermutter. Früher war ich fromm, dann war ich in der FDJ und der Partei, heute glaube ich nur noch, was ich im Portemonnaie habe. (VJ, 298)

Ainsi le roman met-il en œuvre une dialectique entre catastrophes individuelles et collectives. Le jour de septembre 93 sur les hauteurs du pont Köhlbrandbrücke (Kapitel 2-Arbeit) est mis en relation avec la catastrophe collective du 11.09.01, à nouveau intitulé Kapitel 2 - Arbeit, qui, par le truchement médiatique, est perçue des hauteurs du ChileHaus, les tours induisant un parallèle architectural frappant. Quelle relation établir entre préoccupations individuelles et événements collectifs ?

Die sehr disparaten Informationen, die jetzt wie Gesteinsbrocken nach einer Vulkaneruption zwischen ihnen herumlagen (Streß und McKinsey, Köhlbrandbrücke und neuer Job, Kai und mangelnder Sex) mußten in den nächsten Tagen beiseitegeräumt werden, und das war eine so ermüdende Arbeit, daß daneben alles, was sonst noch in der Welt geschah, nebensächlich erschien: daß Steffi Graf am Samstag die US-Open gewonnen hatte, daß sich Rabin und Arafat mit Clinton im Weißen Haus getroffen hatten, das waren Fernsehbilder, die zu anderen Zeiten einen Teil der verfügbaren Emotionen absorbiert hätten, jetzt aber bei Charly nicht nur auf Desinteresse stießen, sondern fast schon auf verächtliche Empörung. (VJ, 81-82)

50. - Michael Kleeberg, Ebd., p. 269: „Ich habe die DDR immer gehasst, von Ulbricht über Stecher und Sparwasser bis Guillaume“.

51. - Michael Kleeberg, Ebd., p. 271: „Die von den Russen gehirngewaschenen Deutschen. Die falschen Deutschen“.

52. - Michael Kleeberg, Ebd., p. 280 : „Meine Frau ist die Tochter eines SEDMannes, scheiße“.

53. - Michael Kleeberg, Ebd., p. 283 : „,dieser Phenolgeruch, der in jener Wohnung aus den Wänden und dem Boden schwitzt und den man zunächst einfach für DDR-Muff hält". 
Si la rencontre historique entre Clinton et Arafat l'avait laissé indifférent quelques années plus tôt, Charly assiste à l'effondrement des Twin Towers en suivant attentivement les cours de la bourse en temps réel. Les courbes de la bourse, les graphiques et les diagrammes sont une modalité de l'écriture de l'Histoire immédiate. Les diagrammes de l'économie en ligne sont intégrés dans la texture du roman. L'écriture de l'Histoire se fait sous nos yeux, sur l'écran, via des diagrammes, rappelant qu'au $\mathrm{XXI}^{\mathrm{e}}$ siècle l'écriture du présent est médiatisée. Les courbes de la bourse permettent à Charly d'envisager les scénarios possibles du futur pour le cours à venir du caoutchouc. Face au surgissement de l'événement, le seul récit qui permet de donner un sens au présent immédiat est la langue économique. Pour le protagoniste de Vaterjahre, la courbe de la bourse devient une forme de récit capable d'élucider le présent.

Le passage du dernier chapitre consacré au surgissement de l'événement global illustre ainsi l'imaginaire du présent, dominé par le flux d'informations en continu et obsédé par lui-même. Le manque de distance propre à cette écriture de l'Histoire immédiate est mis en perspective par l'insertion de citations de l'Edda, ce récit de la mythologie nordique. Alors que Charly et ses collaborateurs observent les avions percuter les tours, l'insertion d'extraits de l'Edda vient, à l'instar d'un chœur d'une tragédie antique, mettre en perspective le(s) récit(s) du 11 septembre.

Charly stellte sich auch vor den Fernseher und sah wie einen Vogel, der schwarz durchs Bild wischt, den Schemen eines Flugzeugs, es verschwand hinter dem rauchenden Turm, dann links der Feuerball, eine brennende Blüte, die sich im Zeitraffer nach oben und unten entfaltet, Kronblätter, Kelchblätter. Eine lodernde, sich selbst zerfressende, im Rauch sich auflösende, aufsteigende und niederfallende Todesblüte, die sich im Verblühen in einen feuerspeienden Rachen verwandelt.

Vom Himmel schwinden die heiteren Sterne.

Glutwirbel umwühlen den allnährenden Weltbaum.

Die heiße Lohe beleckt den Himmel.

„... ähh wir können im Moment nichts über die Hintergründe dieses Anschlags sagen..." (VJ, 456-457)

L'intertextualité place l'événement immédiat dans la lignée des catastrophes qui ont secoué l'humanité depuis sa naissance. Les récits du 11 septembre apparaissent ainsi comme des versions, réécritures, d'un même mythe, celui de la catastrophe naturelle. Dans l'ouvrage consacré à sa poétique, Michael Kleeberg considère que le discours de la césure sur le 11 septembre est « exagéré » 54 . Il souligne que « ce qui est déter-

54. - Michael Kleeberg: „Ich finde zum Beispiel die vermeintliche Epochenbedeutung von 9/11 stark übertrieben“. In: Johannes Birgfeld, note 1, p. 53. 
minant, c'est le récit démesuré que les États-Unis produisent à partir de cet événement, et que l'ensemble du monde occidental a adopté »55.

S'inscrivant à l'opposé des discours de la césure liés au 11 septembre, la construction de ce chapitre (re)place l'événement dans la continuité d'un temps plus long et dans une perspective plus large que la vision myope inhérente au récit médiatique. Le 11 septembre apparaît comme une autre catastrophe à replacer dans la longue histoire des catastrophes qui ont frappé l'humanité : les guerres du $\mathrm{XX}^{\mathrm{e}}$ siècle sur un « continent traumatisé » lors de la visite sur le cimetière allemand de Langemarck, les idéologies comme le communisme qui ont frappé les «centaines de milliers de personnes torturées et brisées ainsi que les millions d'autres prises en otage »56 et bien sûr « les victimes des nazis » ${ }^{57}$. Les images en boucle de l'effondrement des Twin Towers que les collaborateurs de Charly Renn scrutent d'un air médusé ne disent rien ni de la complexité de l'événement d'un point de vue géopolitique ni de la souffrance humaine inhérente à cette catastrophe. En revanche, cette représentation, elle, est révélatrice de la mise en récit du présent ; telle est, à n'en pas douter, l'ambition de Michael Kleeberg dans ce chapitre, qui précise, dans l'ouvrage poétologique Michael Kleeberg im Gespräch, que « l'appréciation des changements décisifs et importants dans le monde sont une question de perspective, temporelle comme spatiale $»^{58}$. La perspective de Charly est celle d'un marchand de caoutchouc et d'un père de famille. L'événement se réduit à une valeur marchande et individuelle. Ce qui prime, c'est la courbe des ventes du caoutchouc. De retour chez lui, l'effondrement des tours jumelles n'est plus que l'arrière-fond, littéralement, des adieux à Bella. La famille s'est rassemblée sur le canapé, devenu « une version idyllique du radeau de la Méduse »59 pour évoquer ensemble les bons moments avec la chienne.

Aber wenn Bella sich gut fühlen soll, sagte die verständige Luisa, dann muss auch der Fernseher laufen wie sonst, sonst fragt sie sich, warum es hier so still ist.

Charly nickte ihr zu - ganz genau, Stille, pietätvolle Stille musste auf jeden Fall vermieden werden, und Heike griff nach der Fernbedienung

55. - Michael Kleeberg : „Entscheidend ist das monumentale ,Narrativ', das die USA daraus stricken, und dem die gesamte westliche Welt gefolgt ist". In: Johannes Birgfeld, note 1, p. 53.

56. - Michael Kleeberg, Vaterjahre, p. 269: „die hunderttausend Gepeinigten und Gebrochenen und die Millionen in Geiselhaft Gehaltenen“.

57. - Michael Kleeberg, Ebd., p. 269: „die Opfer der Nazis“.

58. - Michael Kleeberg: „Die Beurteilung der (wichtigen, entscheidenden) Veränderungen in der Welt ist eine Frage der Perspektive, der zeitlichen wie der räumlichen“, in: Johannes Birgfeld, note 1, p. 54.

59. - Michael Kleeberg, Vaterjahre, p. 478: ,eine idyllische Version des Floßes der Medusa“. 
und schaltete den Fernseher an, und man sah sofort wieder die beiden rauchenden Silbertürme vor dem blauen Himmel. Aber sie stellte den Ton leise, sodass die Kommentare nur ein undeutliches Hintergrundrauschen zu den Erzählungen bildeten, die jetzt beginnen sollten, sozusagen die Dünung des Meeres, über die das Renn'sche Floß dahinsegelte. (VJ, 479)

Michael Kleeberg choisit ici de confronter deux écritures du présent : celle du quotidien familial et celle de l'événement, du «11 septembre [qui, comme l'écrit François Hartog], pousse à la limite la logique de l'événement contemporain qui, se donnant à voir en train de se faire, s'historicise aussitôt et est déjà à lui-même sa propre commémoration : sous l'œil des caméras ${ }^{60}$. Mais ici l'événement s'historicise sous les yeux du chien Bella, qui fixe l'écran de son regard moribond:

Sahen die vertrauten Gesichter und Hände und deren Bewegungen, ein wenig weiter weg die unverständlichen Bewegungen auf dem Fernsehschirm, die rauchenden Silberschemen und ihr Aufschäumen, Niedersinken und Wiederauferstehen, die winzigen, fliegengleichen, fallenden, sich drehenden Körper, die ernsten fremden Gesichter uniformierter und zivil gekleideter Männer. (VJ, 483)

Sous le regard du chien qui « chaque jour renouvelle son présent en faisant table rase ${ }^{61}$, les images du 11 septembre sont un présent absolu, gonflé et insensé. L'événement est saisi sans aucune profondeur.

Dans cette suite au roman Karlmann, Michael Kleeberg relève le défi d'écrire un roman sur le temps qui s'émancipe des contraintes romanesques traditionnelles liées à l'intrigue. Les expérimentations esthétiques et formelles renouent clairement avec les ambitions modernistes, confirmant la quête de l'auteur de poursuivre là où la continuité $\mathrm{a}$ été durablement interrompue, avant la césure de 1933.

Wenn es hierzulande überhaupt einen gemeinsamen Nenner gibt, dann wäre es eher die langsame Rückkehr zur künstlerischen Qualität der Zeit vor 1933, das heißt die Rückgewinnung einer Verfügungsbreite über die künstlerischen Mittel, mit denen auch die Perspektive wächst. Die Zäsur der Nazizeit ist ja kaum dramatisch genug einzuschätzen, und durch die Verwilderung der Seelen und damit auch der Sprache und die tragische Weigerung der im Lande Verbliebenen, die rückkehrwilligen Emigranten zu integrieren, den « Überhang der Tradition », wie 145.

60. - François Hartog, Régimes d'historicité, Paris, Seuil, 2003 et 2012, p. 144-

61. - Michael Kleeberg, Vaterjahre, p. 483 : „täglich erneuerten Tabula-rasaGegenwart". 
Hans Mayer das genannt hat, ist ein künstlerisches Loch entstanden, das, scheint mir, erst in unserer Zeit wieder vollständig zuwächst ${ }^{62}$.

Recréant une temporalité dense et étirée, le roman Vaterjahre laisse converger la totalité de la mémoire humaine, anthropologique, dans la texture du texte qui reproduit une expérience du temps composite qui rappelle une vision kaléidoscopique. Il donne corps non seulement au présent qui, pour reprendre les termes de l'auteur, par nature n'en a pas, mais également à un personnage qui est le produit de son époque, embrassant de son «plasma narratif » toutes ses facettes, "parce que l'évolution humaine est une évolution qui va du typique à l'individuel et puis tend vers l'exemplaire, vers le principe, le social et l'historique ${ }^{63}$. La peinture du présent qui se dessine en filigrane du très ordinaire quotidien de Charly Renn figure une Allemagne à deux visages, une société compartimentée, où les accidents de parcours peuvent conduire à une chute vertigineuse et à une mise au ban de la société. Ainsi le diagnostic du présent opéré par Michael Kleeberg dans le roman Vaterjahre rappelle-t-il à bien des égards les constats de François Hartog qui, avec son concept de présentisme, rappelle notre difficulté, dans la société occidentale post-crise de 2008, à voir au-delà d'un présent obsédé par lui-même et érigé comme seul horizon possible.

62. - Michael Kleeberg, in : Johannes Birgfeld, note 1, p. 91.

63. - Michael Kleeberg, in : Johannes Birgfeld, note 1, p. 89: «weil die menschliche Entwicklung eine vom Typischen zum Individuellen und dann weiter zum Allgemeinen, Prinzipiellen, Gesellschaftlichen und Historischen ist ». 
